\title{
Static and impact fatigue behaviour of borosilicate glass ${ }^{\dagger}$
}

\author{
SOUMEN MAITY and B K SARKAR \\ Central Glass and Ceramic Research Institute, Jadavpur, Calcutta 700032, India
}

\begin{abstract}
Failure of a borosilicate glass as a result of repeated impact has been studied. Impact fatigue study was conducted in an improved pendulum type repeated impact apparatus specially designed and fabricated for determining single and repeated impact strength. For elimination of the effect of humidity, repeated impact tests were carried out under liquid nitrogen. Quasi-static measurements were determined under four-point bending. Using a square waveform as applicable to the present impact tests and fracture mechanics interpretation, the number of cycles to failure during impact fatigue tests were predicted from quasi-static fatigue measurements. It has been shown that repeated impact loading has a deleterious effect on the failure cycles compared to slow stressing. The role of an added mechanical effect during repeated impacts has been suggested in controlling the cyclic fatigue behaviour.
\end{abstract}

Keywords. Static; cyclic; impact; fatigue; glass; atmosphere; liquid nitrogen; stress-corrosion; mechanical effect.

\section{Introduction}

The dependence of fracture stress of silicate glasses on loading parameters is of great technological importance and scientific interest considering the rapid stride in their increasing engineering applications. In ceramic literature, static fatigue has been used to describe the phenomenon of delayed fracture of a ceramic member under a sustained constant load. If the delayed fracture occurs under cyclic loading, it is termed cyclic fatigue. The principal experimental and theoretical results on static fatigue have been summarized (Mould 1967; Wiederhorn 1967; Ritter and Sherbourne 1971; Adams and McMillian 1977). It is generally believed that static fatigue results from a stressdependent chemical reaction between $\mathrm{H}_{2} \mathrm{O}$ vapour and the surface of the glass which causes a pre-existing flaw to grow to the critical dimensions for spontaneous crack propagation.

Although the fatigue behaviour of silicate glasses has been studied extensively, most of the work was conducted with weak specimens which had been abraded in preparation or testing. Results of Gurney and Pearson (1948) suggest that, in glasses under static or cyclic stress, times-to-failure and fatigue limits appear to be approximately same. This view has also been corroborated by later works (Proctor et al 1967; Evans and Fuller 1974; Kawakubo and Komeya 1987) on the observation with the theory that time-based crack growth is merely a function of the stress intensity in a particular environment. On the other hand most of the results for alumina and porcelain indicate some effects of cyclic loading in that the cyclic fatigue life is shorter than the static fatigue life (Krohn and Hasselman 1972; Chen and Knapp 1974; Guiu 1977) and that the apparent threshold stress under cyclic loading is lower than that under static

\footnotetext{
'Paper presented at the poster session of MRSI AGM VI. Kharagpur, 1995
} 
loading (Sedlacek and Halden 1967; Guiu 1977). The above effect was shown to be more severe in case of repeated impact loading (Sarkar and Glinn 1969).

The purpose of this experimental study is to characterize cyclic fatigue behaviour of borosilicate glass under repeated impact loading and compare the results with the predictions based on quasi-static crack growth data. A direct comparison of static and cyclic fatigue results will thus provide some insight into the failure mechanisms in glasses, which will lead to a better mechanistic base for modelling cyclic fatigue and/or static failure in brittle materials.

\section{Experimental}

\subsection{Materials}

Specimens used in the present study were commercially available borosilicate glass rods having a diameter of $5.75 \pm 0.25 \mathrm{~mm}$. Rods of length $60 \mathrm{~mm}$ were cut in a Low Speed Isomet (Buehler Ltd.) using an Isomet saw of grit size $2 \mu \mathrm{m}$. Samples were subsequently annealed at $565^{\circ} \mathrm{C}$ for $30 \mathrm{~min}$ and then furnace cooled to room temperature to eliminate any residual stresses in the sample during the cutting operation. The room temperature properties of the annealed rods as measured were as follows: elastic modulus $63.7 \mathrm{GPa}$ and fracture toughness $0.789 \mathrm{MPam}^{1 / 2}$.

\subsection{Test facility}

Quasi-static tests under atmospheric conditions were carried out in an Instron 1185 electro-mechanical universal testing machine. Specimens were tested under 4-point bending with a minor and major span of $20 \mathrm{~mm}$ and $40 \mathrm{~mm}$ respectively. The specimen loading points were supported by roller bearings to eliminate any frictional effects. Specimens were tested at five different cross-head speeds of $0 \cdot 05,0 \cdot 10,0 \cdot 20,0 \cdot 50$ and $1.00 \mathrm{~mm} / \mathrm{min}$. Breaking strength was calculated using the standard rod formula.

For impact fatigue tests, a swinging pendulum type repeated impact tester was designed and developed based essentially on the principles of Charpy Impact Tester (figure 1). It consisted of a rotating circular disc attached to a horizontal shaft on one end. The circular disc was rotated by a geared pulley system attached to a variable speed motor. To the other end of the horizontal shaft was mounted on ball bearings a pendulum type hammer arm, to the lower end of which was attached a cylindrical hammer. A trigger type mechanism was fixed to one side of the circular disc which contacted the limit switch once during each revolution thus registering the number of impacts. A spring loaded pick-up arm attached to the circular disc carried up the pendulum arm during rotation. Upon contacting with the cam device set at a pre-determined angle, it retracted, allowing the pendulum hammer arm to fall. After each impact the freely hanging hammer was picked up again and again released till the specimen fractured.

Test specimen was held between two tapered conical cups which were screw mounted to the two adjacent platforms. Electrical contact through the specimen was maintained by a streak of graphite running lengthwise. Upon specimen fracture the circuit deactivated thus stopping the machine. A magnetic damping device prevented the hammer from making multiple contacts after each impact during a given revolution, but could be deactivated during single impact studies. 


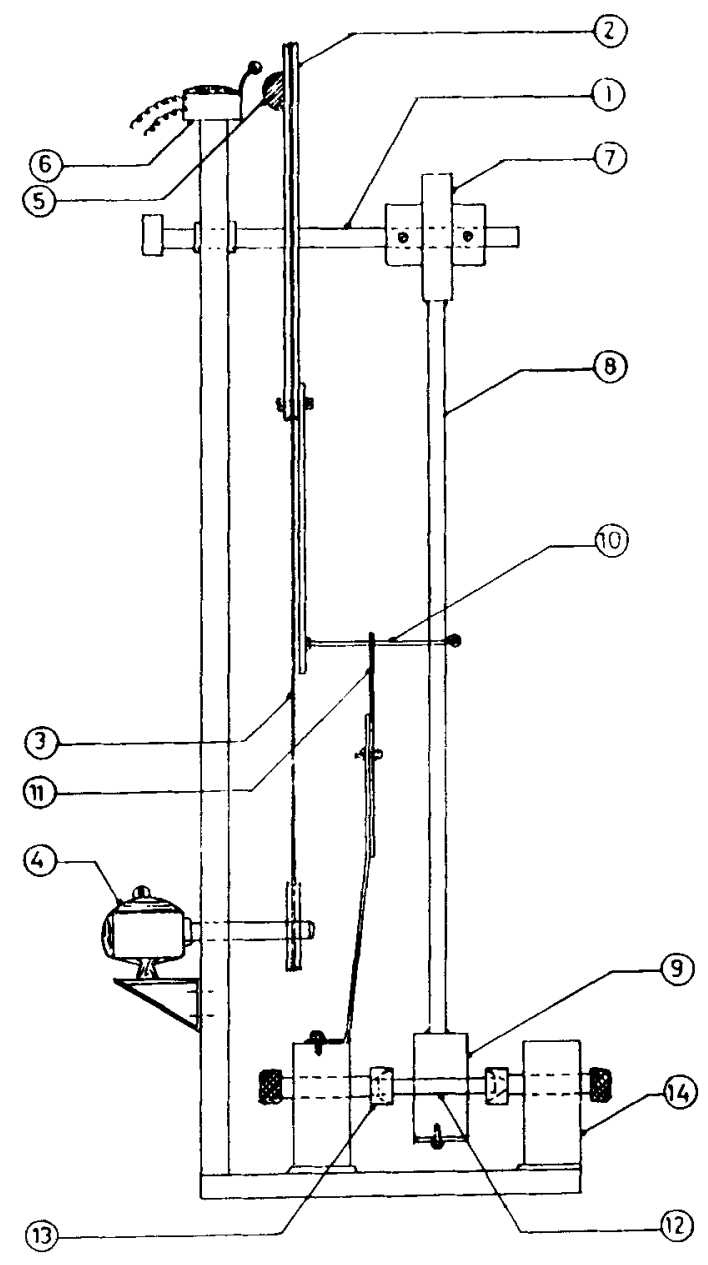

Figure 1. Side elevation of repeated impact fatigue apparatus (1-horizontal shaft; 2 -circular disc; 3-geared pulley; 4-motor; 5-trigger; 6-limit switch; 7-bearing support; 8-pendulum arm; 9-hammer; 10-pick-up arm; 11-cam; 12-specimen; 13-conical grip; 14-specimen support platform).

\subsection{Impact test procedure and loading path}

For repeated impact fatigue tests, a pendulum length of $28.3 \mathrm{~cm}$ and a hammer weight of $253.8 \mathrm{~g}$ was used. Impact energy $(E)$ was calculated according to the formula

$$
E=m g h(1-\cos \theta)
$$

where $m$ was the mass of the hammer, $g$ the gravitational constant, $h$ the length of the pendulum arm and $\theta$ the angle of hammer release.

Energy losses due to windage, friction and 'toss' were determined using a broken specimen placed between the grips. The hammer was released from various angles in the range of $80^{\circ}-10^{\circ}$ and the final angle of swing noted. Difference between the two swings converted to energy were the total losses incurred. The impact energy imparted 
to the specimen was thus obtained after subtracting the energy losses from the impact energy $E$ at $\theta$ angle of release. Impact energy on the specimen was converted to stress $(\sigma)$ according to the formula (Timoshenko and Young 1968)

$$
\sigma=\frac{P}{A}+\sqrt{\left\{\frac{P^{2}}{A^{2}}+\frac{2 Y P h}{A l}\right\}}
$$

where $A, l$ were the cross-sectional area and length of the sample respectively, $h$ the height of the impact after accounting for the energy losses. $P$ the applied load and $Y$ the elastic modulus.

Single blow impact strength was determined by impacting from a high angle. For repeated impact tests, the angle was set at decreasing intervals of $5^{c}$ below the angle required for single impact. It should be mentioned that complete rebound after each impact was eliminated by a magnetic damper device. Fatigue testing was carried out for at least five specimens at each stress level. Tests were terminated beyond $3 \times 10^{4}$ impacts.

Impact fatigue tests were performed with a stress ratio $R=0\left(R=\sigma_{\min } / \sigma_{\max }\right)$ under an impact loading waveform as shown in figure 2 . The testing frequency for impact loading using the rectangular waveform was set at $8 \mathrm{impacts} / \mathrm{min}$.

Repeated impact tests were also performed under liquid nitrogen for suppressing any effect of humidity contributing to static fatigue phenomenon.

\section{Results and discussion}

\subsection{Impact fatigue data}

Results of impact fatigue tests with the rectangular loading waveform has been shown in figure 3 as a plot of maximum applied stress against number of impacts to failure. Each point represents a single specimen tested to failure. Arrowed points indicate that failure did not occur when the test was terminated. Impact strength was plotted as a single cycle to failure. From the figure fatigue lives were observed to increase with decrease in applied stress, indicating that reduction in life during repeated impacts far

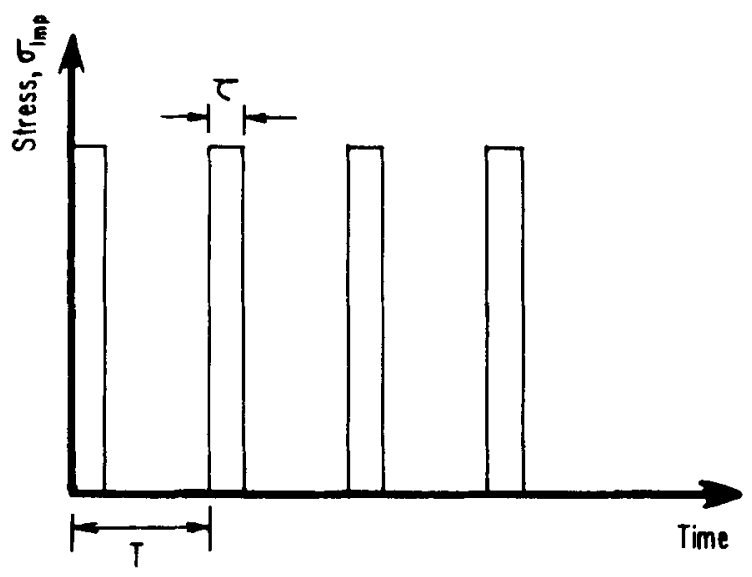

Figure 2. Schematic diagram of loading waveform used in the present impact fatigue tests. 


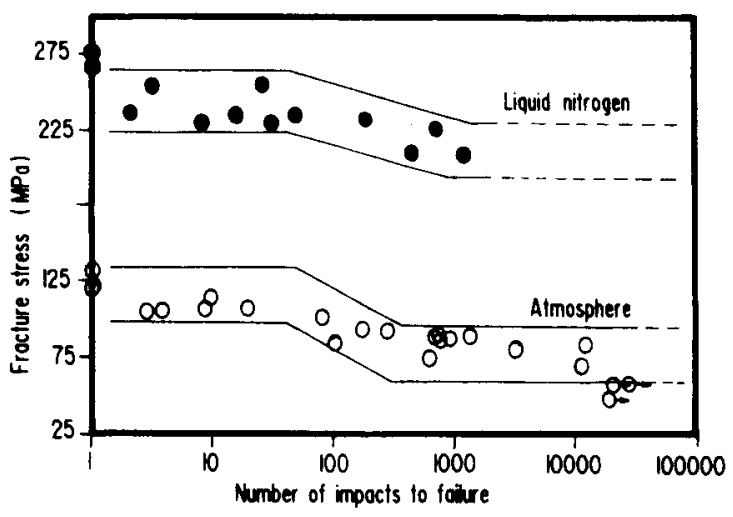

Figure 3. Comparison of impact fatigue lifetimes measured under liquid nitrogen and normal laboratory atmosphere (relative humidity $92 \%$ : temperature $30^{\circ} \mathrm{C}$ ). Arrowed points indicate unbroken samples.

below the threshold strength is a real phenomenon in the case of pristine borosilicate glasses.

Fatigue tests under liquid nitrogen (figure 3) showed that there was a definite beneficial effect on fatigue life compared to tests under atmospheric conditions. This indicated that impact fatigue crack growth in normal atmosphere was accelerated by moisture assisted stress corrosion at the crack tip. When the atmospheric effects were retarded, impact fatigue was purely a manifestation of mechanical factors. Thus cyclic fatigue under impact loading conditions was a mixed mode mechanical and moisture assisted delayed cracking.

\subsection{Comparison of static and impact fatigue}

Subcritical crack growth in ceramics is usually described by a power law of the form (Wiederhorn 1974)

$$
V=\mathrm{d} a / \mathrm{d} t=A K_{1}^{n}=A(Y \sigma \sqrt{a})^{n},
$$

where $V$ is the crack velocity during crack propagation, $a$ the crack size, $t$, the time, $A$ and $n$ are material constants which are functions of temperature and environments, $K_{1}$ the stress intensity factor, $Y$, a geometric factor and $\sigma$ the applied stress. Assuming that the failure mechanisms of static and cyclic are identical and fully time dependent (Lin and Socie 1991), the lifetimes for cyclic loading can be predicted from the measurement of quasi-static fatigue data.

The applied stress $\sigma$. during cyclic loading can be generally expressed as

$$
\sigma=\sigma_{\max } f(t)
$$

where $\sigma_{\max }$ is the maximum applied stress. The failure time $t_{\mathrm{f}}$, can be obtained from integration of (1) as follows

$$
a_{\mathrm{f}}^{(2-n) / 2}-a_{\mathrm{i}}^{(2-n) / 2}=[(2-n) / 2] A Y^{n} \sigma_{\max }^{n} \int_{0}^{t}[f(t)]^{n} \mathrm{~d} t .
$$




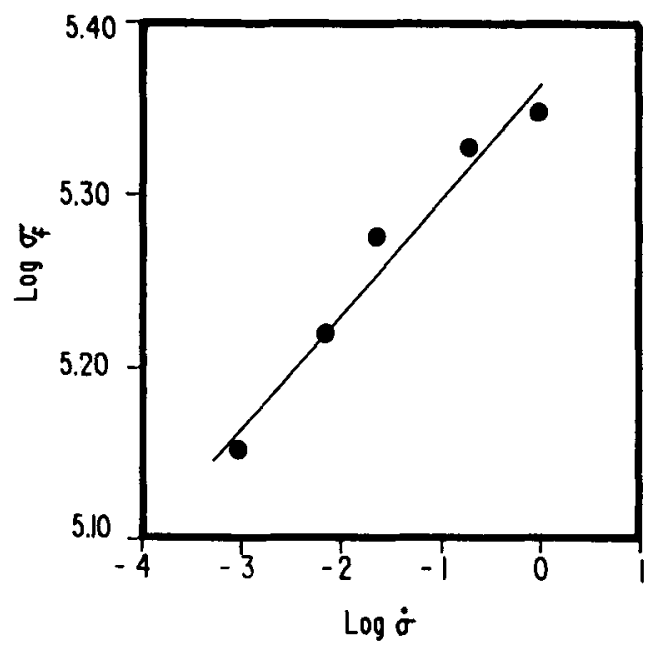

Figure 4. Strength-stress rate relationship under quasi-static loading.

where $a_{\mathrm{i}}$ and $a_{\mathrm{f}}$ are the initial and final crack sizes. In most cases, $a_{\mathrm{f}} \gg a_{\mathrm{i}}$ and $n \gg 1$; then the first term in the left hand side of (3) can be neglected compared to the second term, and simplified as

$$
a_{\mathrm{i}}^{(2-n) / 2}=[(2-n) / 2] A Y^{n} \sigma_{\max }^{n} \int_{0}^{t_{i}}[f(t)]^{n} \mathrm{~d} t .
$$

For the present fatigue tests performed under an impact loading waveform with a period $\tau$, the impact lifetime, $t_{\text {imp }}$ can also be obtained by simplifying (4) as follows

$$
t_{\mathrm{imp}} \cong[2 /(n-2)] A^{-1} Y^{-n} a_{\mathrm{i}}^{(2-n) / 2} \sigma_{\max }^{-n} \tau\left\{\int_{0}^{\tau}[f(t)]^{n} \mathrm{~d} t\right\}^{-1}
$$

In (5) the number of impacts to failure $N_{\mathrm{f}}$ can be had from $t_{\text {imp }}=N_{\mathrm{f}} \tau$ with an approximation that $N_{\mathrm{f}} \gg 1$.

The periodical function, $f(t)$ during each cycle for the impact loading waveforms used in this study is expressed as

and

$$
f(t)=1 \quad(\text { for } 0 \leqslant t \leqslant \tau)
$$

$$
f(t)=0 \quad(\text { for } \tau \leqslant t \leqslant T),
$$

where $T$ is the period of the cyclic waveform.

Substituting (6) in (5), number of impacts to failure is obtained from

$$
N_{\mathrm{f}} \cong[2 /(n-2)] A^{-1} Y^{-n} a_{\mathrm{i}}^{(2-n) / 2} \sigma_{\max }^{-n} \tau^{-1} \text {. }
$$

The crack growth exponent, $n$, has been determined to have a value of 14.01 by the linear regression analysis of the strength-stress rate relationship plotted in figure 4 . The parameter $A$ was also evaluated from the same data using the relation (Wiederhorn 1974)

$$
A=\frac{\sigma^{n+1} Y^{2} K_{1 c}^{n-2}(n-2)}{2(n+1) \dot{\sigma} \sigma_{1 c}^{n-2}}
$$




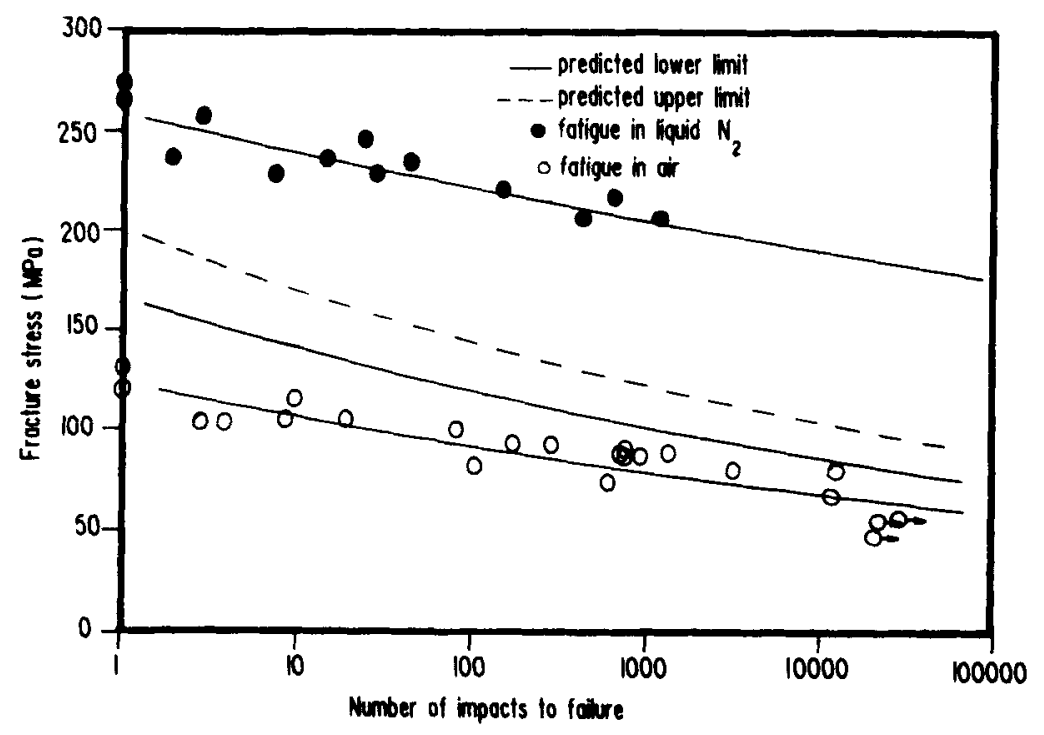

Figure 5. Comparison of measured impact fatigue lifetimes with predicted values from quasi-static tests. Arrowed points indicate unbroken samples.

where inert strength $\sigma_{1 \mathrm{c}}$ was measured as $270 \cdot 3 \mathrm{MPa}$ with corresponding $a_{\mathrm{i}}=6.80 \mu \mathrm{m}$. As the experimental scatter of fatigue data in static and cyclic loading has been an important factor in the confusion of possible 'true' cyclic fatigue of brittle materials in some of the literature, the scatter in the static fatigue data is also taken into consideration in the prediction of impact fatigue lifetime.

The predicted impact fatigue lifetime was thus plotted along with measured lifetime as shown in figure 5 . The predicted upper and lower bound of impact fatigue lifetime was based on the upper and lower bound of static fatigue data. If there was no apparent cyclic effect on the crack growth rate, the measured impact fatigue lifetimes should have been comparable with those predicted from the quasi-static data. But from figure 5 it was evident that the measured impact fatigue lifetimes were much below the lower limit of the predicted values, and the deviations increase with increase in applied stress. Measured impact lifetimes were approximately an order of magnitude lower than the predicted values.

The above comparison indicated that there was apparently a deleterious effect on the failure cycles during repeated loading compared to static one. It was therefore implicit that cyclic loading by repeated impacts was more detrimental than static loading in the borosilicate glass tested under the conditions in this investigation. In absence of a dislocation assisted plastic flow in the present material, possible cause of fatigue failure during repeated impacts was envisaged by the intermittent development of residual stresses during successive impacts followed by its dissipation partly during progress of the crack front.

\section{Conclusions}

The failure of borosilicate glass has been determined by the time taken for pre-existing cracks to grow to a critical size. It has been demonstrated that under repeated impact 
loading below the threshold fracture stress borosilicate glass exhibits progressive endurances with decrease in applied stress. Under identical atmospheric conditions cyclic loading with repeated impacts of short durations provides a detrimental effect on fatigue lifetime in comparison to static loading at the same maximum stress. Testing under liquid nitrogen definitely had a highly beneficial effect on the cyclic lifetime indicating an active role of stress corrosion mechanism. In absence of a dislocation assisted plastic flow, a cumulative residual stress induced at each impact has been suggested as a possible cause of fatigue failure during repeated impacts of borosilicate glass.

\section{Acknowledgements}

The authors are grateful to Dr K K Phani, Shri S Chatterjee, D Basu and M Roychoudhury for help rendered in carrying out this investigation. Financial assistance from CSIR, New Delhi, in the form of a research fellowship is also gratefully acknowledged.

\section{References}

Adams R and McMillan P W 1977 J. Mater. Sci. 12643

Charles R J 1958 J. Appl. Phys. 291549

Chen C P and Knapp W J 1974 in Fracture mechanics of ceramics (eds) R C Bradt et al (New York: Plenum Press) vol. 2 pp. 691-707

Evans A G and Fuller E R 1974 Metall. Trans. 527

Guiu F 1977 J. Mater. Sci. Letts. 131357

Gurney C and Pearson S 1948 Proc. R. Soc. London Ser. A192 537

Kawakubo T and Komeya K 1987 J. Am. Ceram. Soc. 70400

Krohn D A and Hasselman D P H 1972 J. Am. Ceram. Soc. 55208

Lin C J and Socie D F $1991 \mathrm{~J}$. Am. Ceram. Soc. 741511

Mould R E 1967 in Fundamental phenomena in the material sciences (New York: Plenum Press) pp. 119-149

Proctor B A, Whitney I and Johnson J W 1967 Proc. R. Soc. London Ser. A297 534

Ritter J E and Sherbourne C L 1971 J. Am. Ceram. Soc. 54601

Sarkar B K and Glinn T G J 1969 J. Mater. Sci. 4951

Sedlacek R and Halden F A 1967 in Structural ceramics and testing of brittle materials(eds) S J Acquaviva and S A Bortz (New York: Gordon and Breach Science Publ.) pp. 211-220

Timoshenko S P and Young D H 1968 in Elements of strength of materials (New York: Litton Educational Publ. Inc.)

Wiederhorn S M 1967 J. Am. Ceram. Soc. 50407

Wiederhorn S M 1974 in Fracture mechanics of ceramics (eds) R C Bradt et al (New York: Plenum Press) Vol. 2, pp. $623-646$ 